\title{
Las fitohormonas una pieza clave en el desarrollo de la agricultura
}

\section{The plant hormones, an important component of the agriculture development}

\author{
Borjas-Ventura Ricardo* ${ }^{(D)}$, Julca-Otiniano Alberto ${ }^{(D)}$, Alvarado-Huamán Leonel ${ }^{\circ}$
}

\section{Datos del Artículo}

Departamento Académico de Fitotecnia. Facultad de Agronomía.

Universidad Nacional Agraria La Molina.

Av. La Molina s/n.

La Molina, Perú

Telf. +51 01614-7800

ajo@lamolina.edu.pe

lealvarado@lamolina.edu.pe

*Dirección de contacto:

Departamento Académico de Fitotecnia.

Faculad de Aronomí

Universidad Nacional Agraria La Molina.

Av. La Molina s/n.

La Molina, Perú

Telf. +51 01614-7800

Ricardo Borjas-Ventura

E-mail address: rborjas@lamolina.edu.pe

\section{Palabras clave:}

Agricultura,

seguridad alimentaria,

reguladores de crecimiento,

hormonas.

\section{J. Selva Andina Biosph.} 2020; 8(2):150-164.

ID del artículo: 092/JSAB/2020

\section{Historial del artículo.}

Recibido abril 2020

Devuelto julio 2020.

Aceptado septiembre 2020

Disponible en línea, noviembre 2020

\section{Editado por:}

Selva Andina

Research Society
2020. Journal of the Selva Biosphere ${ }^{\circledR}$. Bolivia. Todos los derechos reservados.

La agricultura es una de las actividades más importantes del ser humano. Esta actividad no solo aporta riqueza a los países, sino también, es de vital importancia para la seguridad alimentaria. Con la finalidad de atender una demanda de alimentos cada vez en aumento, se ha venido usando diferentes insumos agrícolas, entre ellos destacan los reguladores de crecimiento, los mismos, que están relacionados con el crecimiento y desarrollo de las plantas. Estos reguladores de crecimiento contienen hormonas cuyo rol en diferentes procesos metabólicos de las plantas ha sido ampliamente estudiado, sin embargo, la información sistematizada sobre su uso práctico en la agricultura es aun escaso. Esta información es clave puesto que puede ser usada por diversos investigadores agrarios, para potenciales aplicaciones en nuevos cultivos. En este contexto, esta revisión busca sistematizar la información sobre el descubrimiento, síntesis y uso práctico de reguladores de crecimiento en la agricultura.

\section{Abstract}

Agriculture is one of the most important activities of humans. This activity not only brings wealth but also is key to the food safety of countries. In order to meet the increasing demand for food, the farmers have been using different agricultural supplies as growth regulations (GR) that are related to plant growth and development. These GR content hormones whose role in different metabolic processes of the plants are widely studied; however, the systematized information on the practical use of plant hormones in agriculture is still scarce. This information is key since it can be used by many agricultural researchers in order to find potential applications in new cultures. In this context, this review seeks to systematize the information on the discovery, synthesis, and practical use of PGR in agriculture.

\section{Keywords:}




\section{Introducción}

La agricultura es una de las actividades más importantes de la sociedad actual, especialmente en países en vías de desarrollo, por ejemplo, en Perú, esta actividad representó el 4\% del Producto Bruto Interno (PBI) y empleó a cuatro millones de personas en el $2019^{1}$, además de ser una actividad clave en la seguridad alimentaria, al suministrar una cantidad importante de alimentos para la nutrición humana ${ }^{2}$.

Ante una población cada vez más numerosa ${ }^{3}$, y para atender mayores demandas de alimentos, la agricultura se está tecnificando cada vez más. En la actualidad existen una serie de insumos que mejoran el crecimiento y desarrollo de los cultivos. Entre los insumos usados frecuentemente, por los agricultores, se encuentran los biorreguladores o reguladores de crecimiento (RC) que son definidos como: “com puestos naturales o sintéticos que afectan los procesos metabólicos", y que en última instancia pueden mejorar la productividad y calidad de las cosechas ${ }^{5}$.

Entre los principales compuestos que regulan los procesos metabólicos de las plantas tenemos a las hormonas vegetales (HV), que hasta el momento son diez: Auxinas, citoquininas, giberelinas, ácido abscísico, etileno, ácido salicílico, poliaminas, ácido jasmónico, brasinoesteroides, y estrigolactonas) ${ }^{6}$, siendo las cinco primeras denominadas "hormonas clásicas" cuyos descubrimientos se remontan a más de medio siglo atrás ${ }^{7-11}$.

Estas hormonas clásicas tienen diversas funciones en el desarrollo de las plantas, así, la auxina está relacionada con la división y elongación celular ${ }^{12}$, las giberelinas en la maduración del polen y el desarrollo de flores, frutos y semillas ${ }^{7}$, el etileno en la expansión y división celular ${ }^{13}$, el ácido abscísico (ABA) regula la abertura y cierre de estomas, mientras que las citoquininas están envueltas en la división celular y la morfogénesis de los tejidos ${ }^{11}$.
El párrafo anterior denota un amplio conocimiento sobre el papel de las HV en el crecimiento y desarrollo de las plantas. Este conocimiento ha hecho que la aplicación de reguladores de crecimiento hormonales ( $\mathrm{RCH})$ (sintéticos) sea de uso común en cultivos hortícolas ${ }^{14,15}$, plantas ornamentales ${ }^{16,17}$, cultivos de uso industrial ${ }^{18,19}$ y árboles forestales ${ }^{20}$. Sin embargo, actualmente existen pocos trabajos de revisión y sistematización de datos sobre el uso de hormonas en la agricultura ${ }^{21,22}$, especialmente en español y en revistas científicas. Este tipo de información es relevante ya que puede ser usada por estudiantes, personal técnico y científicos, que quieran llevar a cabo experimentos revisando para obtener nuevos datos o replicar los experimentos mencionados en otros cultivos. En este sentido, esta revisión busca sistematizar la información del descubrimiento, síntesis y uso práctico de las hormonas en la agricultura.

\section{Desarrollo}

Las hormonas vegetales son una pieza clave en el crecimiento y desarrollo de las plantas, aunque también se encuentran vinculados a su defensa ${ }^{23}$. Aunque descubiertas en el siglo pasado, con el pasar del tiempo y con la mejora de las técnicas químicas, se han ido descubriendo muchas más características que han permitido desarrollar insumos para la agricultura, mejorando de esta forma los rendimiento de los cultivos. En esta sección repasamos brevemente a las hormonas clásicas y sus usos en agricultura.

Auxinas y su aplicación en la agricultura. El nombre de esta hormona deriva del griego "auxein" que significa "crecer". Uno de los experimentos clave que ayudó al descubrimiento de substancias auxinas fue el desarrollado por Went en 1928 (con el coleoptilo de avena). Posteriormente, se han ido descu- 
briendo compuestos auxínicos, siendo el primero de ellos del ácido indol acético (IAA) ${ }^{24}$. Además, se encuentra en mayores concentraciones en zonas de crecimiento como los ápices del vástago o de la raíz ${ }^{25}$.

La síntesis de esta hormona se lleva a cabo por una vía independiente del triptófano y por otra dependiente del mismo, siendo esta última la más importante. En el caso de la vía dependiente del triptófano, existen cuatro rutas metabólicas que llevan los nombres de los compuestos intermediarios más importantes: i) 3-indol acetamida (IAM), ii) 3-indol acetaldoxima (IAOx), iii) triptamina (TAM) y ácido 3-indol pirúvico (IPA) $)^{6,25-27}$ (figura 1).

\section{Figura 1 Rutas de síntesis de auxinas que dependen del triptófano}

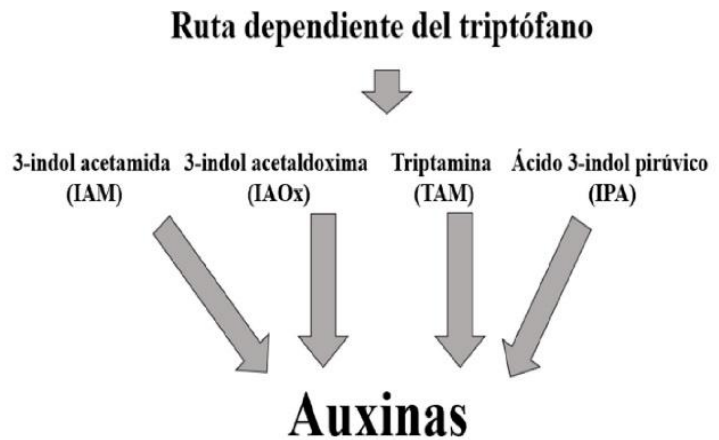

Existen diferentes tipos de auxinas en las plantas: IAA, ácido fenilacético (PAA), ácido indol butírico (IBA) y ácido indol propiónico (AIP) ${ }^{25}$. Asimismo, la auxina generalmente, en las plantas, se encuentra en forma conjugada. Esta forma conjugada (desactivada) es importante porque le permite a la planta poder almacenar auxinas, y liberarlas cuando sea necesario, por ejemplo, durante la madurez de la semilla es almacenado cantidades importantes de auxina conjugada, que posteriormente será usada en la germinación $^{28}$. También podemos mencionar auxinas sintéticas como ácido naftalen acético (ANA), dicamba $2,4-\mathrm{D}$ y $2,4,5-\mathrm{T}^{25}$.
Respecto al transporte de auxinas, esta puede ser de dos tipos: Larga distancia (transporte rápido) y a corta distancia (transporte lento). En la primera de ellas, la auxina se transporta por los conductos floemáticos (siguiendo el movimiento Fuente-dreno), mientras que en la segunda el movimiento de la auxina es de célula a célula, siendo esta última importante para la formación del eje embrionario, la respuesta a los tropismos, la filotaxia, dominancia apical y la morfogénesis de la raíz, flor y fruto ${ }^{6}$.

El transporte a corta distancia o transporte polar ${ }^{6}$ requiere una serie de proteínas transportadoras y de energía para poder desarrollarse (transporte activo), y tanto en el tallo como en la raíz es basipétala ${ }^{28}$, aunque puede haber un movimiento lateral (observado en el tallo). Una vez que la auxina llega a la base del tallo (desde el ápice superior), la hormona pasa a la raíz llegando hasta el ápice y posteriormente regresa a la base del tallo (movimiento basipétalo $^{29}$ (figura 2).

Figura 2 Movimiento polar y no polar de las auxinas

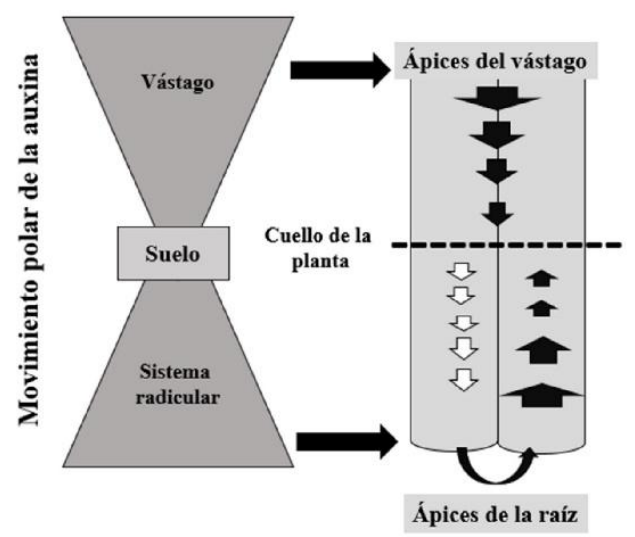

Las auxinas se encuentran relacionadas con la elongación y división celular, la diferenciación de tejidos y la respuesta la luz y la gravedad (tropismos), disminución de la abscisión de los órganos, induce la formación de raíces adventicias, inducción de la diferenciación vascular y el estímulo de la dominancia apical ${ }^{12}$. 
La aplicación de RC, con base en auxinas, en la agricultura, se concentra básicamente en aprovechar su capacidad como enraizante, así puede usarse para la propagación de diversos materiales genéticos, por ejemplo en frutales, plantas ornamentales o plantas de uso industrial como el café y cacao (tabla 1).

Tabla 1 Aplicación de reguladores de crecimiento a base de auxinas en la agricultura

\begin{tabular}{|c|c|c|c|c|c|}
\hline Especies & Prod & Dosis & Variable & Rpta & Referencia \\
\hline Rhododendron simsii & $\begin{array}{l}\text { AIB } \\
\text { ANA }\end{array}$ & $8000 \mathrm{~mL} \mathrm{~L}^{-1}$ & Enraizamiento de estacas & SR & 30 \\
\hline Fragaria $x$ anannasa & AIB & $0.15 \%$ & $\begin{array}{l}\text { Altura de planta } \\
\text { Número de hojas } \\
\text { Longitud de raíz }\end{array}$ & - & 31 \\
\hline Agave tequilana & $\begin{array}{l}\text { ANA } \\
2.4 \mathrm{D}\end{array}$ & $3 \mathrm{mg} \mathrm{L}^{-1}$ & Callo embriogénico & + & 32 \\
\hline Eustona grandiflorum & AIB & $\begin{array}{c}250 \mathrm{ppm} \\
1000 \mathrm{ppm}\end{array}$ & Enraizamiento estacas & + & 33 \\
\hline Malpighia sp & $\begin{array}{c}\text { ANA } \\
\text { AIB }\end{array}$ & $\begin{array}{l}4000 \mathrm{mg} \mathrm{k}^{-1} \\
6000 \mathrm{mg} \mathrm{k}^{-1}\end{array}$ & Enraizamiento en acodo aéreo & + & 34 \\
\hline Cheilocostus speciosus & ANA & $500 \mathrm{mg} \mathrm{L}^{-1}$ & Enraizamiento esquejes & + & 35 \\
\hline Rosa sp & $2.4 \mathrm{D}$ & $0.5 \%$ & Formación de raíces en estacas & + & 16 \\
\hline Citrullus lanatus & $2.4 \mathrm{D}$ & $200 \mathrm{mg} \mathrm{L}^{-1}$ & Brix & + & 14 \\
\hline
\end{tabular}

Giberelinas y su aplicación en la agricultura. En 1926, el investigador Eiichi Kurosawa descubría, en el hongo Gibberella fujikuroi, una substancia que incrementaba de forma significativa la altura de las plantas, incluso en detrimento del rendimiento. Para 1950, se determinaba la síntesis de esta hormona en plantas $^{7}$, la misma que fue denominada Giberelina ${ }^{25}$. Las giberelinas (GAs) son diterpenoides que generalmente se sintetizan en los mismos lugares de acción $^{36,22}$. Su síntesis involucra los plastidios, el retículo endoplasmatico y el citosol de la célula, asimismo, todos los tipos de giberelinas son sintetizados en la ruta del ácido mevalonico ${ }^{22}$. En los plastidios ocurre la formación del ent-kaurene, el cual se dirige al retículo endoplasmatico y ahí es transformado en $\mathrm{GA}_{12}$. En el citoplasma, $\mathrm{GA}_{12}$ se transforma en $\mathrm{GA}_{1}$ y $\mathrm{GA}_{4}$ (formas activas) ${ }^{37}$.

Los diferentes tipos de giberelinas se diferencian por número de carbono y por la presencia del grupo hidroxilo. Las GAs bioactivas son $\mathrm{GA}_{1}, \mathrm{GA}_{3}, \mathrm{GA}_{4} \mathrm{y}$ $\mathrm{GA}_{7}^{22}$. De los mencionados destaca el $\mathrm{GA}_{3}$ que es una de los más usadas en la agricultura, por ejemplo en la producción de uvas de mesa sin semilla y en el aumento de la calidad de cítricos y peras. En el caso de $\mathrm{GA}_{4}$ y $\mathrm{GA}_{7}$ son más difícil de separar de los fermentos de G. fujikuroi ${ }^{4}$.

En cuanto a los inhibidores de la síntesis de giberelinas, tenemos los siguientes: cloruro de clormequat, cloruro de mepiquat, ancymidol, paclobutasol, flurpirmidol, uniconazol, tebuconazol, daminozide, 16, 17 dihidroxigiberelinas y metconazol ${ }^{4}$.

Esta hormona se encuentra relacionada a la germina ción de semillas, elongación del tallo, expansión de la hoja, maduración del polen y el desarrollo de flores, frutos y semillas ${ }^{7}$. Aunque, en función de su dosis y del estado fenológico de la planta, los biorre guladores en base a giberelinas pueden promover el crecimiento del vástago (por ejemplo en plantas arbóreas), la germinación de semillas e incrementar el rendimiento entre otras funciones (tabla 2).

Etileno y su aplicación en la agricultura. Conocida también como hormona del estrés (ya que responde a factores ambientales de tipo biótico y abiótico) ${ }^{43}$, esta hormona puede estar en concentraciones de 0.01-1.0 $\mathrm{ppm}^{8}$. A finales del siglo XVIII, Dimitry Neljubow notó que el epicotilo de arveja se etiolaba, engrosaba y que se flexionaba horizontalmente (triple acción) al estar cerca de fuentes de iluminación. 
Este investigador determino que el gas usado en las fuentes de iluminación contenía el compuesto de- nominado etileno. En 1934, se descubrió que la planta biosintetizaba etileno $\left(\mathrm{C}_{2} \mathrm{H}_{4}\right)^{8}$.

Tabla 2 Aplicación de reguladores de crecimiento a base de giberelinas en la agricultura

\begin{tabular}{|c|c|c|c|c|c|}
\hline Especies & Prod & Dosis & Variable & Rpta & Referencia \\
\hline Solanum lycopersicum & $\mathrm{GA}_{3}$ & $100 \mathrm{mg} \mathrm{L}^{-1}$ & Tolerancia a Ralstonia solanacearum & + & 38 \\
\hline Eucalyptus urophylla x Eucalyptus camaldulensis & $\mathrm{GA}_{3}$ & $100 \mathrm{mg} \mathrm{L}^{-1}$ & Crecimiento de plantas & + & 20 \\
\hline Swietenia macrophylla & PROGIBB & $400 \mathrm{mg} \mathrm{L}^{-1}$ & Crecimiento de plantas & + & 39 \\
\hline Diferentes especies frutales de clima templado & $\mathrm{GA}_{3}$ & $\begin{array}{l}\text { En función } \\
\text { de la especie }\end{array}$ & $\begin{array}{l}\text { Inhibición de floración, frutos parte- } \\
\text { nocarpicos, tamaño y raleo de fruto }\end{array}$ & + & 40 \\
\hline Psidium cattleianum & $\mathrm{GA}_{3}$ & $400 \mathrm{mg} \mathrm{L}^{-1}$ & Promoción de la germinación & + & 41 \\
\hline Plukenetia volubilis & $\mathrm{GA}_{3}$ & $\begin{array}{c}60-100 \mathrm{mg} \\
\mathrm{L}^{-1}\end{array}$ & Rendimiento & + & 19 \\
\hline Psidium guajava & $\mathrm{GA}_{3}$ & $50 \mathrm{ppm}$ & Tamaño de semilla & - & 38 \\
\hline Kalankloe longiflora & $\mathrm{GA}_{3}$ & $\begin{array}{l}100-200 \mu \mathrm{g} \\
\text { planta }^{-1}\end{array}$ & $\begin{array}{c}\text { Número de inflorescencia y número } \\
\text { de flores }\end{array}$ & + & 17 \\
\hline Rubus glaucus & $\mathrm{GA}_{3}$ & $1000 \mathrm{ppm}$ & Porcentaje de germinación & + & 42 \\
\hline
\end{tabular}

SR: Sin respuesta, +: positiva, -: negativa

La síntesis del etileno se desarrolla en tres pasos: En primer lugar, la metionina es convertida en SAM (S-adenosyl-L-methionine), luego el SAM es transformado en ACC (1-aminocyclopropane-1-carboxy lic acid) con la ayuda de la enzima ACC sintasa (ACS), en el ciclo de Yang. Posteriormente, la ACC es transformado en etileno con la ayuda de la enzima ACC oxidasa ${ }^{8,13}$. Es importante mencionar que la enzima ACS cumple una función reguladora (de su mayor o menor activación dependerá la cantidad de etileno sintetizado) ${ }^{44}$ (figura 3 ).

Figura 3 Ruta metabólica de la síntesis de etileno

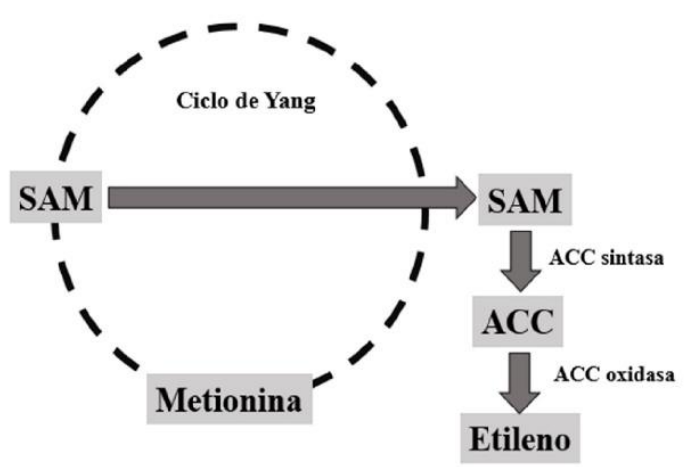

Como fue mencionado en el párrafo anterior, la síntesis de etileno se encuentra relacionada a la metionina, en este sentido, el $80 \%$ de la metionina celular es transformada en Ado-metionina (que posteriormente será convertido en ACC), Además, la célula puede reutilizar la metionina, reciclándola de la Ado-metionina ${ }^{45}$. Por otra parte, la Ado-metio nina es precursor de la espermidina y de la espermi$\mathrm{na}^{46}$.

La ACS requiere energía para su síntesis, esta característica envuelve la presencia de $\mathrm{O}_{2}$, mientras que para su activación esta enzima requiere la presencia tanto de $\mathrm{O}_{2}$ como de $\mathrm{CO}_{2}$. En tejidos vegetativos inmaduros (no climatéricos) la síntesis de $\mathrm{C}_{2} \mathrm{H}_{4}$ es inhibida por $\mathrm{C}_{2} \mathrm{H}_{4}$, mientras que en tejidos maduros climatéricos la síntesis es promovida por $\mathrm{C}_{2} \mathrm{H}_{4}$ (a este fenómeno se le conoce como efecto autocatalítico $)^{47}$.

Esta hormona se encuentra involucrada en la expansión y división celular (crecimiento de la planta) ${ }^{13}$, desarrollo de las flores, maduración del fruto, la respuesta de al estrés biótico y abiótico ${ }^{48}$, además, acelera la senescencia y la abscisión razón por la cual también se le conoce como hormona del envejecimiento $^{49}$ (tabla 3 ).

El movimiento del etileno es por difusión y en general su lugar de producción está cerca de su lugar de acción, por tal motivo, al parecer, no son necesarios transportadores proteicos, de hecho, hasta la fecha 
no se tiene información de ellos ${ }^{8}$. Esta hormona

puede ser usada para mejorar la calidad del fruto (color, sabor y aroma) o para el raleo de los mismos entre otros usos (tabla 4).

Tabla 3 Efectos del etileno en la agricultura (Saltveit ${ }^{47}$ )

\begin{tabular}{|c|c|c|}
\hline Promueve & Inhibe & Efectos negativos \\
\hline La madurez de frutos climatéricos & La síntesis de etileno en frutos no climatéricos & Acelera la senescencia \\
\hline Síntesis de antocianinas en la maduración de frutos & Transporte de auxinas & Ablandamiento excesivo de frutos \\
\hline Destrucción de la clorofila y amarillamiento & Elongación de la raíz y del vástago & Estimula la pérdida de clorofila \\
\hline Germinación de semillas & & Promueve la absición de hojas y flores \\
\hline \multicolumn{3}{|l|}{ Formación de raíces adventicias } \\
\hline \multicolumn{3}{|l|}{ Respiración } \\
\hline \multicolumn{3}{|l|}{ Iniciación de la floración en bromealiacias } \\
\hline Absición y senescencia & & \\
\hline
\end{tabular}

Tabla 4 Aplicación de reguladores de crecimiento a base de etileno en la agricultura

\begin{tabular}{|c|c|c|c|c|c|}
\hline Especies & Prod & Dosis & Variable & Rpta & Referencia \\
\hline Spondias cytherea & Etefon & $\begin{array}{l}1.50 \mathrm{ppm} \\
2.25 \mathrm{ppm}\end{array}$ & Desverdeamiento del fruto & + & 50 \\
\hline Capsicum аппиит & Etileno & $10 \mu \mathrm{mol} \mathrm{L}-1$ & Caída de flores & + & 51 \\
\hline Coffea arabica & Etefon & $130 \mathrm{ml} \mathrm{L}^{-1}$ & $\begin{array}{l}\text { Calidad organoléptica del } \\
\text { café }\end{array}$ & SR & 18 \\
\hline Ficus carica & Etefon & $\begin{array}{l}500 \mathrm{ppm} \\
1000 \mathrm{ppm}\end{array}$ & Firmeza de frutos & - & 52 \\
\hline Averrhoa carambola $L$. & Etefon & $15 \mathrm{ml} \mathrm{L}^{-1}$ & Madurez y color de fruta & + & 54 \\
\hline Sorghum bicolor & Etefon & $400 \mathrm{ppm}$ & Contenido de azúcar & + & 55 \\
\hline Vaccinium virgatum & Etefon & $600 \mathrm{mg} \mathrm{L}^{-1}$ & Madurez de fruta & + & 56 \\
\hline Hevea brasiliensis & Etefon & $3 \%$ & Rendimiento de latex & + & 57 \\
\hline
\end{tabular}

SR: Sin respuesta; +: positiva; -: negativa

Ácido abscísico y su aplicación en la agricultura. El Ácido abscísico (ABA) es una hormona vegetal descubierta en los años 60’s. En relación a su biosíntesis ${ }^{10,60}$, este proceso se lleva cabo en dos lugares diferentes de la célula: plastídios (por ejemplo cloroplastos y amiloplastos) y citosol. Además, la ruta de síntesis del ABA es La vía dependiente del ácido mevalónico. Esta ruta comienza con la conversión del $\beta$-caroteno, zeaxantina y la anterazantina en trans-violaxantina o en su isómero la Neoxantina (en el plastídio). Estas transformaciones son mediadas por la enzima zeaxanthin epoxidase (ZEP). Una vez formada la violaxantina, ella puede transformarse en 9-cis-violaxanthin o 90-cis-neoxanthin, ambas moléculas darán origen a Xanthoxin (con la ayuda de la enzima 9-cis-epoxy carotenoid dioxygenase
(NCED)) que migrará desde el plastídio hasta el citosol. Una vez en el citosol, la Xanthoxin sufrirá dos transformaciones sucesivas para originar el ABA.

Respecto al transporte, inicialmente se creía que el ABA se sintetizaba solo en las raíces, sin embargo, actualmente se sabe que la síntesis de esta hormona se produce también en hojas ${ }^{61}$, incluso el ABA producido en las hojas puede afectar significativamente el desarrollo de la raíz ${ }^{62}$. El transporte a corta distancia (entre célula y célula) lo puede realizar de forma activa (con ayuda de algunos transportadores) o pasiva ${ }^{63}$.

Esta hormona tiene una influencia muy grande sobre el crecimiento y desarrollo de la planta, de hecho, ella participa en la germinación, división celu- 
lar, modula la arquitectura de las raíces, regula cierre y abertura de estomas, promueve la dormancia de las semillas, respuesta a estreses como la salinidad, la sequía, la radiación y el ataque de patógenos 9,10 . Respecto a su uso en agricultura, esta hormona puede ser usada en diversos cultivos para aumentar la tolerancia a diversos estreses abióticos o en algunos casos para mejorar la cantidad de azucares solubles en frutales como la vid (tabla 5).

Tabla 5 Aplicación de reguladores de crecimiento a base de ácido abscísico en la agricultura

\begin{tabular}{|c|c|c|c|c|c|}
\hline Especies & Prod & Dosis & Variable & Rpta & Referencia \\
\hline Vitis vinifera & $\mathrm{ABA}$ & $40 \mathrm{~g} 100 \mathrm{~L}^{-1}$ & $\begin{array}{c}\text { Índice de coloración, contenido } \\
\text { de antocianinas }\end{array}$ & + & 64 \\
\hline Vitis vinifera & $\mathrm{ABA}$ & $\begin{array}{l}20{\mathrm{~g} 100 \mathrm{~L}^{-1}} \\
40 \mathrm{~g} 100 \mathrm{~L}^{-1}\end{array}$ & Grados Brix & + & 64 \\
\hline Pennisetum purpureum & $\mathrm{ABA}$ & $100 \mu \mathrm{mol} \mathrm{L}^{-1}$ & Tolerancia al frio & + & 65 \\
\hline Populus euphratica & $\mathrm{ABA}$ & $100 \mu \mathrm{M}$ & $\begin{array}{l}\text { Disminución de toxicidad por } \\
\text { cádmio y actividad de enzimas } \\
\text { antioxidantes }\end{array}$ & + & 66 \\
\hline Bidens pilosa & $\mathrm{ABA}$ & $40 \mu \mathrm{mol} \mathrm{L}{ }^{-1}$ & Extracción de cadmio & + & 67 \\
\hline Solanum lycopersicum & $\mathrm{ABA}$ & $\begin{array}{l}500 \mathrm{mg} \mathrm{L}^{-1} \\
50 \mathrm{mg} \mathrm{L}^{-1} \\
5 \mu \mathrm{mol} \mathrm{L}^{-1}\end{array}$ & $\begin{array}{l}\text { Contenido de glucosa y fructo- } \\
\text { sa }\end{array}$ & + & 68 \\
\hline Pisum sativum & $\mathrm{ABA}$ & $\begin{array}{l}10 \mu \mathrm{mol} \mathrm{L}^{-1} \\
20 \mu \mathrm{mol} \mathrm{L}\end{array}$ & Acumulación de cadmio & - & 69 \\
\hline Arabidopsis thaliana & $\mathrm{ABA}$ & $0.1-0.5 \mu \mathrm{M}$ & Acumulación de cadmio & - & 70 \\
\hline Triticum aestivum & $\mathrm{ABA}$ & $10 \mu \mathrm{mol} \mathrm{L}{ }^{-1}$ & Tolerancia a la falta de agua & + & 71 \\
\hline Solanum lycopersicum & $\mathrm{ABA}$ & $\begin{array}{l}500 \mathrm{mg} \mathrm{L}^{-1} \\
50 \mathrm{mg} \mathrm{L}^{-1}\end{array}$ & Influjo de Ca hacia los frutos & + & 72 \\
\hline Cucumis sativus & $\mathrm{ABA}$ & $200 \mu \mathrm{mol} \mathrm{L}^{-1}$ & Tolerancia al frio & + & 73 \\
\hline
\end{tabular}

Citoquininas y su aplicación en la agricultura. Las citoquininas son definidas como: "substancias que promueven la división celular y ejercen otras funciones similares a la Kinetina"74. La historia de las citoquininas empieza desde los albores del siglo XX cuando se descubría una sustancia que estimulaba la división celular, pero no fue hasta los años 50's cuando se descubrió la verdadera identidad de aquella misteriosa substancia ${ }^{11}$. Fue en el esperma de arenque donde Miller y colaboradores descubren lo que denominarían Kinetina $^{75}$, posteriormente (1961) se descubrió que las plantas poseían sus propias citoquininas siendo la más importante la Zeatina (aislada del maíz) $^{11}$.

La citoquinas son derivadas de adeninas y clasificadas como: isoprenoides, citoquininas derivadas de adeninas con sustituyentes aromáticos y las difenilureas sintéticas. En el primer grupo podemos encontrar la Zeatina y el iP, mientras que en el segundo grupo podemos encontrar a los topolins ${ }^{76,77}$. La con- centración de esta hormona en el tejido vegetal es del orden de $\mathrm{pmol} / \mathrm{g}$ de materia fresca ${ }^{78}$. Asimismo, la homeostasis de esta hormona se lleva a cabo por procesos como: biosíntesis, activación, degradación y conjugación ${ }^{77}$.

Como producto de la ruta del mevalonato (MVP)/Methylerythritol Phosphate (MEP) se forma el dimethylallyl diphosphate (DMAPP), el cual, con la ayuda de phosphate-isopentenyltransferase (IPT), transfiere un resto isopentenil al ATP, ADP o al AMP formando iPRTP, iPRDP y iPRMP respectivamente. En este punto existen dos caminos: 1) que tanto el iPRTP y el iPRDP se transformen en iPRMP, y que este último de origen al $\mathrm{N}^{6}-\Delta^{2}$ isopentenyl)adenine (iP) directamente o con la participación de la enzima N-ribohydrolase (NRH). 2) que iPRTP, iPRDP y, iPRMP sufran hidroxilación formando tZRTP, tZRDP y tZRMP (denominados $t Z$ nucleotidos). Es importante anotar que tZRTP y tZRDP pueden interconvertirse en tZRMP $^{78}$. 
El tZRMP formado tiene dos caminos: 1) dar origen trans-zeatin (tZ) (directamente o con ayuda de la enzima 5'-monophosphate phosphoribohydrolases (LOGs)), o 2) transformarse en DZRMP. Este último puede dar origen a dihydrozeatin (DZ) (direc- tamente o con ayuda de la enzima LOGs). La formación de (cZ) sigue principalmente la ruta metabólica MVP (en el citosol de la célula) con la participación de $\mathrm{LOGs}^{78}$.

Tabla 6 Aplicación de reguladores de crecimiento a base de citoquinina en la agricultura

\begin{tabular}{|c|c|c|c|c|c|}
\hline Especies & Prod & Dosis & Variable & Rpta & Referencia \\
\hline Vaccinium corymbosum & Trans-Zeatina & $2 \mathrm{mg} \mathrm{L}^{-1}$ & $\begin{array}{l}\text { Número de brote y altura } \\
\text { de planta en cultivo in } \\
\text { vitro }\end{array}$ & + & 85 \\
\hline Zinnia elegans & $\begin{array}{c}\text { BAP (6- } \\
\text { bencilaminopurina) }\end{array}$ & $50 \mu \mathrm{M}$ & Severidad de $B$. cinérea & - & 86 \\
\hline Perezia pinnatifida & BAP & $1 \mathrm{mg} \mathrm{L}^{-1}$ & $\begin{array}{l}\text { Número de brote en } \\
\text { cultivo in vitro }\end{array}$ & + & 87 \\
\hline Passiflora quadrangularis & Zeatina & $2 \mathrm{ppm}$ & $\%$ de germinación & SR & 88 \\
\hline Aegle marmelos & BAP & $50 \mathrm{ppm}$ & $\begin{array}{l}\text { Brotación temprana en } \\
\text { portainjerto, número de } \\
\text { hojas }\end{array}$ & + & 89 \\
\hline Capsicum аппит & BAP & $\begin{array}{c}50 \mu \mathrm{M} \\
100 \mu \mathrm{M}\end{array}$ & $\begin{array}{l}\text { Número de frutos por } \\
\text { planta }\end{array}$ & - & 90 \\
\hline Capsicum аппит & BAP & $\begin{array}{c}50 \mu \mathrm{M} \\
100 \mu \mathrm{M}\end{array}$ & $\begin{array}{c}\text { Contenido de capsicina } \\
\text { de frutos }\end{array}$ & + & 90 \\
\hline Capsicum аппит & BAP & $\begin{array}{c}50 \mu \mathrm{M} \\
100 \mu \mathrm{M}\end{array}$ & Ácido ascórbico de frutos & + & 90 \\
\hline Solanum lycopersicum & Trans-Zeatina & $25 \mathrm{ppm}$ & $\begin{array}{l}\text { Número de flores por } \\
\text { planta; número total de } \\
\text { frutos }\end{array}$ & + & 91 \\
\hline Oriquedea Dendrobium & BAP & $200 \mathrm{mg} \mathrm{L}^{-1}$ & Ancho del pétalo & + & 92 \\
\hline Ananas comosus & Zeatina & $\begin{array}{l}24 \mathrm{ppm} \\
48 \mathrm{ppm}\end{array}$ & Peso del fruto & SR & 93 \\
\hline Epipremnum aureum & BAP & $5 \mathrm{mg} \mathrm{L}^{-1}$ & Peso seco de la raíz & + & 94 \\
\hline
\end{tabular}

El iP, tZ, DZ y cZ son las citoquininas más abundantes en las plantas, mientras que otras las podemos encontrar en menor cantidad como los Topolins (del grupo de las citoquininas derivadas de adeninas con sustituyentes aromáticos) ${ }^{79,77}$.

Esta hormona es sintetizada en diferentes partes de las plantas como raíces, flores hojas jóvenes, etc. ${ }^{79}$. Aunque, los tipos de citoquininas varían en función de la especie y del órgano estudiado, por ejemplo, la cis-Zeatina se encuentra mayormente en papas, arroz, maíz y muchas legumbres, mientras que la Dihidroxizeatina la podemos encontrar en semillas dormantes, sirviendo probablemente como fuente de citoquinina para el posterior desarrollo de la plántu$1 \mathrm{a}^{74}$. Asimismo, la presencia de esta hormona vegetal varía en función de la especie, del estado fenológico y de las condiciones ambientales donde se desarrollan las plantas ${ }^{74}$.
El transporte de la citoquinina es realizado en el xilema y en floema (desde la raíz hasta la parte aérea y viceversa) (transporte a larga distancia) ${ }^{80,77}$, sin embargo, tanto en el floema como en el xilema podemos encontrar diferentes tipos de Zeatinas ${ }^{81}$. Por otra parte, la tZ es sintetizada principalmente en la raíces, motivo por el cual, la podemos encontrar mayormente en el xilema, mientras que el iP es sintetizada principalmente por la parte aérea de las plantas razón por la cual la encontramos mayormente en el floema ${ }^{78}$.

Es importante anotar que las citoquininas pueden existir en firmas inactivas (conjugadas) como iPRMP/iPR, tZRDP/tZR, DZRMP/DZR, y cZRMP/cZR ${ }^{82}$, que pueden ser activadas y dar sus formas libres (iP, tZ, DZ y cZ). La conjugación (desactivación reversible o irreversible) es realizada con azucares o aminoácidos ${ }^{78}$. 
Entre las funciones de esta fitohormona, se encuentra su participación en la defensa contra estreses abióticos $^{83}$ y bióticos ${ }^{84}$, en la propagación in vitro de plantas y en el aumento de rendimiento (tabla 6).

\section{Conclusiones y perspectivas futuras}

Existe un amplio consenso sobre la síntesis y funciones de hormonas como auxinas, citoquininas, giberelinas, ácido abscísico y etileno, las cuales son usadas frecuentemente en una agricultura intensiva. Estos reguladores de crecimiento son usados en la agricultura para aprovechar su capacidad como enraizantes, en la germinación de semillas, maduración de frutos, tolerancia a diversos tipos de estrés y en el incremento de la producción. Sin embargo, el uso de biorreguladores hormonales está notablemente limitado a los tipos de cultivos, es decir, faltan más investigaciones en cultivos potencialmente importantes, asimismo, se sugiere investigar cómo los biorreguladores pueden ayudar a la planta a superar condiciones de estrés abiótico especialmente el estrés hídrico y térmico, siendo ambos variables ambientales cambiantes con el cambio climático.

\section{Fuente de financiamiento}

Al ser una revisión no hubo fuente financiamiento.

\section{Conflictos de intereses}

La investigación presente no tiene conflictos de intereses.

\section{Agradecimientos}

Los autores agradecen a la Universidad Nacional Agraria La Molina.

Aspectos éticos
Los autores declaran que el manuscrito se desarrolló utilizando cuidadosamente el aporte intelectual de estudios en la literatura y reconociéndolos mediante el respectivo citado de autores y fuentes.

\section{Literatura Citada}

1. Sector agrario aporta $5.4 \%$ del PBI y emplea a más de 4 millones de peruanos [Internet]. Andina-Agencia Peruana de Noticias. 2020 [citado 3 febrero 2020]. Recuperado a partir de: https:// andina.pe/agencia/noticia-sector-agrario-aporta-

54-del-pbi-y-emplea-a-mas-4-millones-peruanos776467.aspx

2. Agua, seguridad alimentaria y agricultura [Internet]. Autoridad Nacional del Agua. 2020. [citado 10 enero 2020]. Recuperado a partir de: https:// www.ana.gob.pe/portal/gestion-del-conocimiento -girh/agua-y-seguridad-alimentaria

3. United Nations Department of Public Information. Crecimiento a un ritmo menor, se espera que la población mundial alcanzará 9700 millones en 2050 y un máximo de casi 11000 millones alrededor del 2100: Informe de la ONU [internet]. [New York]: Departamento de Comunicaciones Globales de las Naciones Unidas; 2019 [citado 3 de febrero de 2020]. Recuperado a partir de: https://population.un.org/wpp/Publications /Files/WPP20 19_PressRelease_ES.pdf

4. Rademacher W. Chemical regulators of gibberellins status and their application in plant production. En: Rademacher W, editor. Annual Plant Review Online. Annual Plant Reviews book series, Volume 49: The Gibberellins. Wiley Online Library; 2017;49:359-403. DOI: https://doi.org/ 10. 1002/9781119312994.apr0541

5. Gollagi SG, Lokesha R, Dharmpal S, Sathish BR. Effects of growth regulators on growth, yield and quality of fruits crops: A review. J Pharmacog phytochem 2019;8(4):979-81. 
6. Garay Arroyo A, de la Paz Sánchez M, García Ponce B, Álvarez Buylla ER, Gutiérrez C. La homeostasis de las auxinas y su importancia en el desarrollo de Arabidopsis thaliana. Rev Educ Bioquím 2014;33(1):13-22.

7. Takehara S, Ueguchi-Tanaka M. Gibberellin. En: Hejátko J, Hakoshima T, editors. Plant Structural Biology: Hormonal Regulations. Switzerland: Springer Nature; 2018. p. 83-95. DOI: https://doi. org/10.1007/978-3-319-91352-0_6

8. Chang C. Q\&A: How do plants respond to ethylene and what is its importance?. BMC Biol 2016;14:1-7. DOI: https://doi.org/10.1186/s129 15-016-0230-0

9. Finkelstein R. Abscisic acid synthesis and response. En: Finkelstein R, editor. The Arabidopsis Book: American Society of Plant Biologists; 2013;11:e0166. DOI: https://doi.org/10.1199/tab. $\underline{0166}$

10.Sah SK, Reddy KR, Li J. Abscisic acid and abiotic stress tolerance in crop plants. Front Plant Sci 2016;7:571. DOI: https://doi.org/10.3389/fpls. 2016.00571

11.Citokinins [Internet]. The International Plant Growth Substances Association. [citado 3 de febrero de 2020]. Recuperado a partir de: https:// pages.wustl.edu/ipgsa/cytokinins

12. Vega Celedón P, Canchignia Martínez H, González M, Seeger M. Biosíntesis de ácido indol-3acético y promoción del crecimiento de plantas por bacterias. CulTrop 2016;37(Suppl 1):33-9.

13.Dubois M, Van den Broeck L, Inzé D. The pivotal role of ethylene in plant growth. Trends Plant Sci 2018;23(4):311-23. DOI: https://doi.org/10. 1016/j.tplants.2018.01.003

14. Cavalcante R, Tavares A, Mendes F, Cerqueira F, Nascimento I. Características pós-colheita de frutos partenocárpicos de melancia obtidos com o uso de 2,4-D. Pesqui Apl Agrotec 2017;10(1):517. DOI: https://doi.org/10.5935/PAeT.V10.N1.05
15. Rodrigues F, Pacheco Nunes AC, Costa Carvalho $\mathrm{DD}$, Ribeiro MC. Induction of tolerance to bacterial wilt in hybrids of tomatoes by application of gibberellin. Rev Ciênc Agrovet 2018;17(1):5460. DOI: https://doi.org/10.5965/2238117117120 $\underline{18054}$

16.De la Cruz Castillo J, Mejía Coico F, Mostacero León J, López Medina E, Gonza Carnero A. Efecto de la concentración del 2,4-dichloro phenoxyacetic acid (2,4-D) en el enraizamiento de estacas de Rosa sp., rosa silvestre, en condiciones de invernadero. Rev Indes 2014;2(1):3743. DOI: https://doi.org/10.25127/indes.201401. $\underline{004}$

17.Coelho LL, Fkiara A, Mackenzie KK, Müller R, Lütken H. Exogenous application of gibberellic acid improves flowering of Kalanchö̈. Hort Science 2018;53(3):342-6. DOI: https://doi.org/10. 21273/HORTSCI12720-17

18. Santos AA, Alves TL, Lemos EF, Ferreira EA, Assis LFS, et al. Qualidade da bebida do café em função do uso de produto a base de etileno na maturação dos frutos [internet]. [Viçosa]: Universidad Federal de Viçosa; 2017. [citado 26 de febrero de 2020]. Recuperado a partir de: http://www.sbicafe.ufv.br/handle/123456789/962 $\underline{1}$

19.Pezo M, Márquez Dávila K, Solis R. El ácido giberélico incrementa el rendimiento de plantas adultas de sacha inchi (Plukenetia volubilis). Scientia Agropecuaria 2019;10(4):455-60. DOI: https://doi.org/10.17268/sci.agropecu.2019-04.01

20.Amaro CL, Cunha SD, Grupioni PHF, Sousa PV de, Abadia KLD, Barros IB, et al. Análise do crescimento de mudas de Eucalyptus sp submetida a diferentes doses de giberelina. AgriEnvironmental Sci 2017;3(1):24-9. DOI: https://doi.org/ 10.30612/agrarian.v13i48.7990

21.Sebastian K, Arya MS, Reshma UR, Anaswara SJ, Thampi S. Impact of plant growth regulators 
on fruit production. Int J Curr Microbiol App Sci 2019;8(2):800-14. DOI: https://doi.org/10.20546/ ijcmas.2019.802.092

22.Ghosh S, Halder S. Effect of different kinds of gibberellin on temperate fruit crops: A review. J Pharm Innov 2018;7(3):315-9.

23.Wang C, Liu Y, Li S, Han G. Insights into the origin and evolution of the plant hormones signaling machinery. Plant Physiol 2015;167:87286. DOI: https://doi.org/10.1104/pp.114.247403

24.Enders TA, Strader LC. Auxin activity: Past, present, and future. Am J Bot 2015;102(2):18096. DOI: https://doi.org/10.3732/ajb.1400285

25.Jordan M, Casaretto J. Hormonas y reguladores de crecimiento: Auxinas, Giberelinas y Citocininas. En: Squeo FA, Cademil L, editores. Fisiología Vegetal [Internet]. Universidad de La Serena. La Serena, Chile; 2006. p. 1-28. Recuperado a partir de: http://www.exa.unne.edu.ar/biologia/ fisiologia. vegetal/Auxinasgiberelinasycitocininas .pdf

26.Di DW, Zhang C, Luo P, An CW, Guo GQ. The biosynthesis of auxin: how many paths truly lead IAA?. Plant Growth Regul 2016;78:275-85. DOI: https://doi.org/10.1007/s10725-015-0103-5

27.Mashiguchi K, Tanaka K, Sakai T, Sugawara S, Kawaide H, Natsume M, et al. The main auxin biosynthesis pathway in Arabidopsis. Proc Natl Acad Sci USA 2011;108(45):18512-7. DOI: https://doi.org/10.1073/pnas.1108434108

28.Rozov SM, Zagorskaya AA, Deineko EV, Shumny VK. Auxins: Biosynthesis, Metabolism, and transport. Biol Bull Rev 2013;3(4):286-95. DOI: https://doi.org/10.1134/S20790864130400 $\underline{87}$

29. Michniewicz M, Brewer F, Friml J. Polar auxin transport and asymmetric auxin distribution. En: Michniewicz M, Brewer F, Friml J, editors. The Arabidopsis Book: American Society of Plant Biologists; 2007;5:e0108. DOI: https://doi.org/ $\underline{10.1199 / \text { tab. } 0108}$
30.Cabral Feliciana AM, Morais EG, Reis Soares E, Corrêa RM, Gontijo A, Vaz GHB. Influência de auxinas e tamanho de estacas no enraizamento de azaleia (Rhododendron simsii Planch.). Gl Sci Technol 2017;10(1):43-50.

31.Juárez Rosete CR, Aguilar Castillo JA, Bugarín Montoya R, Aburto González CA, Alejo Santiago G. Medios de enraizamiento y aplicación de auxinas en la producción de plántulas de fresa. Cienc Tecnol Agropecuaria 2020;21(1):e1319. DOI: https://doi.org/10.21930/rcta.vol21_num1 art: 1319

32.Ángeles Espino A, Sánchez Mendoza L, Dimas Estrada HE, Ramírez Alvarado D, Gómez Leyva JF. Evaluación de dos auxinas en la inducción de callo embriogénico en vitroplántulas de Agave tequilana Weber variedad azul. Trop Subtrop Agroecosyst 2018;21(2):225-34.

33. Sotomayor Leon EM, Rosas Guerra C, Mazuela P. Propagación vegetativa de Lisianthus (Eustoma grandiflorum RAF) cv abc 2-3 Blue Rim. Idesia 2016;34(5):71-3. DOI: https://doi.org/10. 4067/ S0718-34292016005000030

34.Ramírez Villalobos MC, Segundo Urdaneta Fernández A. Efecto de tratamientos auxínicos en el enraizamiento de dos especies de Malpighia mediante la técnica del acodo aéreo. Past Forr 2017;40(2):96-101.

35.Durango Ballesteros E, Humanez Álvarez A. Enraizamíento de esquejes de caña agria (Cheilocostus speciosus J. Koenig). Rev Colomb Biotecnol 2017;19(2):133-9. DOI: http://doi.org/10.15 446/ rev.colomb.biote.v19n2.70395

36. Martins AO, Nunes Nesi A, Araújo WL, Fernie AR. To bring flowers or do a runner: Gibberellins make the decision. Mol Plant 2018;11(1):4-6. DOI: https://doi.org/10.1016/j.molp.2017.12.005

37.Rizza A, Jones A. The makings of a gradient: spatiotemporal distribution of gibberellins in plant development. Curr Opin Plant Biol 
2019;47:9-5. DOI: https://doi.org/10.1016/j.pbi. $\underline{2018.08 .001}$

38. Rodrigues SCA, Ribeiro JM, Santos CAF. Avaliação preliminar do ácido giberélico na produção de goiaba sem sementes. [Internet]. Empresa Brasileira de Pesquisa Agropecuária Ministério da Agricultura, Pecuária e Abastecimento. Brasilia, Brasil. [citado 26 de febrero de 2020]. Recuperado a partir de: https://www.embrapa.br/ busca-de-publicacoes/-/publicacao/1113595/avalia cao-preliminar-do-acido-giberelico-na-producaode-goiaba-sem-sementes

39. Vale Montilla CC. Análisis de crecimiento de plántulas de caoba (Swietenia macrophylla King) en tratamientos con auxinas y giberelinas bajo condiciones de vivero. Pittieria 2018;42:52-72.

40.Sáez Reyes MN. Usos de giberelinas de síntesis en la fruticultura chilena [tesis licenciatura]. [Santiago]: Universidad de Chile; 2016. [citado 20 de febrero de 2020]. Recuperado a partir de: http://repositorio.uchile.cl/handle/2250/150997

41.Silva M, Silva Oliveira LE, Radaelli C, Porto AH, Junior A. Stratification and use of gibberellin in seed of Psidium cattleianum. Appl Res Agrotec 2018;11(3):121-5. DOI: https://doi.org /10.5935/ PAeT.V11.N3.12

42. Vásquez W, Pupiales P, Viteri P, Sotomayor A, Feican C, Campaña D, et al. Escarificación química y aplicación de ácido giberélico para la germinación de semillas de cultivares de mora (Rubus glaucus Benth). Interciencia 2019;44(3): 161-6.

43.Bakshi A, Shemansky JM, Chang C, Binder BM. History of research on the plant hormone ethylene. J Plant Growth Regul 2015;34(4):809-27. DOI: https://doi.org/10.1007/s00344-015-9522-9 44.Harpaz-Saad S, Yoon GM, Mattoo A, Kieber J. The formation of ACC and competition between polyamines and ethylene for SAM. En: McManus MT, editor. Annual Plant Reviews Vol 44: the
Plant Hormone Ethylene. Wiley Online Library; 2012;44:53-81. DOI: https://doi.org/10.1002/978 $1118223086 . \operatorname{ch} 3$

45.Hu Y, Vandenbussche F, Van Der Straeten D. Regulation of seedling growth by ethylene and the ethylene-auxin crosstalk. Planta 2017;245(3) :467-89. DOI: https://doi.org/10.1007/s00425-0 17-2651-6

46.Jouyban Z. Ethylene biosynthesis. Tech J Engin \& App Sci 2012;1(4):107-10.

47.Saltveit M. Ethylene effects. In: Gross K, YiWang, Saltveit M, editors. The commercial storage of fruits, vegetables, and florist and nursery stocks [Internet]. California: United States Department of Agriculture; 2016. p. 76. Recuperado a partir de: https://www.ars.usda.gov/arsuserfiles /oc/np/commercialstorage/commercialstorage.pdf

48. Khan NA, Khan MIR, Ferrante A, Poor P. Ethylene: A key regulatory molecule in plants. Front Plant Sci 2017;8:1782. DOI: https://doi.org/10. 3389/fpls.2017.01782

49. Schaller GE. Ethylene and the regulation of plant development. BMC Biol 2012;10:9. DOI: https:// doi.org/10.1186/1741-7007-10-9

50.Fiúza Ribeiro MC, de Sousa Lima GM, Mizobutsi GP. Desverdecimento do cajá-manga com o uso do etileno. An Acad Pernamb Ciênc Agron 2019;16(2):67-86.

51.Ribeiro WS, Carneiro C, França CF, Pinto CM, Lima PC, Finger FL, et al. Sensitivity of ornamental pepper to ethylene. Hortico Bras 2019a;37(4):458-63. DOI: https://doi.org/10.1590 /s0102-053620190415

52.Sandoval J, Hernández MS, Herrera AO. Effect of ethephon on the ripening process of the fig (Ficus carica L.). Agron Colomb 2016;34(1 Suppl):S1411-4.

53.Macías Duarte R, Grijalva Contreras RL, Robles Contreras F, López Carvajal A, Núñez Ramírez F. Evaluación de reguladores de crecimiento en 
cebolla para el control de la emisión del tallo floral. Rev Mex Cienc Agríc 2017;8(7):1549-60. DOI: https://doi.org/10.29312/remexca.v8i7.511

54.Le PTQ, Pham MH. The effects of ethephon on the ripening of carambola (Averrhoa carambola L.). Int Food Res J 2018;25(4):1497-501.

55. Yoosukyingsataporn S, Detpiratmongkol S. Effects of ethephon on growth and yield of sweet sorghum (Sorghum bicolor L. Moench) at different growth stages. Iran J Plant Physiol 2019;10(1):2987-97. DOI: https://doi.org/10.22 034/IJPP.2019.670785

56.Wang YW, Malladi A, Doyle JW, Scherm H, Nambeesan SU. The effect of ethephon, abscisic acid, and methyl jasmonate on fruit ripening in rabbiteye blueberry (Vaccinium virgatum). Horticulturae 2018;4(3):24. DOI: https://doi.org/10. 3390/horticulturae4030024

57. Attanayake AP, Kurunanayake L, Nilmini AHRL. Effect of ethephon stimulation on natural rubber latex properties; new insight into ethephon stimulation. J Natl Sci Found 2018;46(2):179-85. DOI: https://doi.org/10.4038/jnsfsr.v46i2.8418

58. Anzanello R, Tedesco A. Ethephon in the chemical thinning of fruits of peach cultivar Chimarrita. Revista Electrônica Científica da UERGS 2020;6(1):66-71. DOI: https://doi.org/10.21674/ 2448-0479.61.66-67

59.Pesteanu A. Effects of ethephon application on color development of "Gala Must" apple. Bull Univ Agric Sci Vet Med Cluj-Napoca Hortic 2017;74(1):26-32. DOI: https://doi.org/10.15835/ buasvmen-hort:12267

60. Vishwakarma K, Upadhyay N, Kumar N, Yadav G, Singh J, Mishra RK, et al. Abscisic acid signaling and abiotic stress tolerance in plants: A review on current knowledge and future perspectives. Front Plant Sci 2107;8:161. DOI: https:// doi.org/10.3389/fpls.2017.00161

61.Sussmilch FC, Brodribb TJ, McAdam SA. What are the evolutionary origins of stomatal responses to abscisic acid in land plants?. J Integr Plant $\mathrm{Bi}$ ol 2107;59(4):240-60. DOI: https://doi.org/10. 1111 /jipb.12523

62.McAdam SA, Manzi M, Ross JJ, Brodribb TJ, Gómez Cadenas A. Uprooting an abscisic acid paradigm: Shoots are the primary source. Plant Signal Behav 2016;11(6):e1169359. DOI: https://doi.org/10.1080/15592324.2016.1169359

63.Dar NA, Amin I, Wani W, Wani SA, Shikari AB, Wani SH, et al. Abscisic acid: A key regulator of abiotic stress tolerance in plants. Plant Gene 2017;11(Pt 2):106-11. DOI: https://doi.org/10. 1016/j.plgene.2017.07.003

64.Pereira Gardin JP, Schumacher RL, Bettoni JC, Petri JL, de Souza EL. Ácido abscísico e etefom: influência sobre a maturação e qualidades das uvas Cabernet Sauvignon. Rev Bras Frutic 2012;34(2):321-7. DOI: https://doi.org/10.1590 /S0100-29452012000200003

65.Wang Y, Zhu M, Yang Y, Alam I, Cheng X, Qin $\mathrm{T}$, et al. Exogenous application of abscisic acid (ABA) enhances chilling tolerance in seedling of napier grass (Pennisetum purpureum Scgum). Agric Sci Technol 2017;18(3):417-23. DOI: https://doi.org/10.16175/j.cnki.1009-4229.2017. $\underline{03.008}$

66.Han Y, Wang S, Zhao N, Deng S, Zhao C, Li N, et al. Exogenous abscisic acid alleviates cadmium toxicity by restricting $\mathrm{cd}^{2+}$ in Populus euphratica cell. J Plant Growth Regul 2016;35(3):827-37. DOI: https://doi.org/10.1007/s00344-016-9585-2

67.Liu L, Ma Q, Lin L, Tang Y, Wang J, Lv X, et al. Effect of exogenous abscisic acid on cadmium accumulation in two ecotypes of hyperaccumulator Bidens pilosa. Environ Prog Suitain Energy 2017;36(6):1643-9. DOI: https://doi.org/10.1002/ ep.12619

68. Barickman TC, Kopsell DA, Sams CE. Abscisic acid impacts tomato carotenoids, soluble sugars, and organic acid. HortScience 2016;51(4):370-6. 
DOI: https://doi.org/10.21273/HORTSCI.51.4. $\underline{370}$

69.Lu Q, Sun G, Liu J, Tang Y. Effect of abscisic acid on growth and cadmium accumulation of pea seedling. En: Lu Q, Sun G, Liu J, Tang Y, editors. Proceedings of the 2018 3rd International Conference on Advances in Materials, Mechatronics and Civil Engineering, ICAMMCE 2018 [Internet]. Advances in Engineering Research; 2018. DOI: https://doi.org/10.2991/icammce-18. 2018.14

70.Fan SK, Fang XZ, Guan MY, Ye YQ, Lin XY, Du ST, et al. Exogenous abscisic acid application decreases cadmium accumulation in Arabidopsis plants, which is associated with the inhibition of IRT1-mediated cadmium uptake. Front Plant Sci 2014;16:712. DOI: https://doi.org/10.3389/fpls. $\underline{2014.00721}$

71.Du YL, Wang ZY, Fan JW, Turner NC, He J, Wang $\mathrm{T}$, et al. Exogenous abscisic acid reduces water loss and improves antioxidant defence, desiccation tolerance and transpiration efficiency in two spring wheat cultivars subjected to a soil water deficit. Functional Plant Biology 2013;40(5):494-506. DOI: https://doi.org/10.10 71/FP12250

72.Barickman TC, Kopsell DA, Sams CE. Exogenous foliar and root application of abscisic acid increase influx of calcium into tomato fruit tissue and decrease the incidence of blossom-end rot. HortScience 2014;49(11):1397-402. DOI: https: //doi.org/10.21273/HORTSCI.49.11.1397

73. Ying Z, Weijie J, Hongjun Y, Xueyong Y. Exogenous abscisic acid alleviates low temperatureinduced oxidative damage in seedling of Cucumis sativus L. Transactions of the Chinese Society of Agricultural Engineering 2012;28(2):221-8. DOI: https://doi.org/10.3969/j.issn.1002-6819.2012.z2. $\underline{038}$
74.Frébort I, Kowalska M, Hluska T, Frébortová J, Galuszka P. Evolution of cytokinin biosynthesis and degradation. J Exp Bot 2011;62(8):2431-52. DOI: https://doi.org/10.1093/jxb/err004

75.Osugi A, Sakakibara H. Q\&A: How do plants respond to cytokinins and what is their importance?. BMC Biol 2015;13:102. DOI: https:// doi.org/10.1186/s12915-015-0214-5

76. Mok DW, Mok MC. Cytokinin metabolism and action. Annu Rev Plant Physiol Plant Mol Biol 2001;52:89-118. DOI: https://doi.org/10.1146/an nurev.arplant.52.1.89

77.Jameson PE, Song J. Cytokinin: a key driver of seed yield. J Exp Bot 2016;67(3):593-606. DOI: https://doi.org/10.1093/jxb/erv461

78. Márquez López RE, Quintana Escobar AO, Loyola Vargas VM. Cytokinins, the Cinderella of plant growth regulators. Phytochem Rev 2019;18(6):1387-408. DOI: https://doi.org/10.10 07/s11101-019-09656-6

79.Hirose N, Takei K, Kuroha T, Kamada Nobusada T, Hayashi H, Sakakibara H. Regulation of cytokinin biosynthesis, compartmentalization and translocation. J Exp Bot 2008;59(1):75-83. DOI: https://doi.org/10.1093/jxb/erm157

80.Schmulling T. Cytokinin. In: Lennarz W, Lane MD, editors. Encyclopedia of Biological Chemistry [Internet]. Berlin: Academic Press/Elsevier Science; 2004. p. 1-7. Recuperado a partir de: https://www.bcp.fu-berlin.de/biologie/arbeitsgrup pen/angenetik/forschungsgruppen/ag_schmue lling/AGSCHMUELLING_pdfs/Cytokinin .pdf

81.Skalický V, Kubeš M, Napier R, Novák O. Auxins and cytokinins-the tole of subcellular organization on homeostasis. Int $\mathrm{J}$ Mol Sci 2018;19(10):3115. DOI: https://doi.org/10.3390/ ojms19103115

82.Kieber JJ, Schaller GE. Cytokinin signalling in plant development. Development 2018;145(4): 
dev149344. DOI: https://doi.org/10.1242/dev. 149344

83. Cortleven A, Leuendorf JE, Frank M, Pezzetta D, Bolt S, Schmülling T. Cytokin action in response abiotic and biotic stress in plants. Plant Cell Environ 2019;42(3):998-1018. DOI: https://doi.org /10.1111/pce.13494

84.Choi J, Choi D, Lee S, Ryu C, Hwang I. Cytokinins and plant immunity: old foes or new friend?. Trends Plant Sci 2011;16(7):388-94. DOI: https://doi.org/10.1016/j.tplants.2011.03.003

85. Arista Bustamante JP, Leiva Espinosa ST, Guerrero Abad JC, Collazos Silva R. Efecto de las citoquininas en la multiplicación in vitro de cuatro variedades de Vaccinium corymbosum a partir de segmentos nodales. Rev Científica UNTRM 2019;1(2):55-62. DOI: https://doi.org/10.25127/ ucni.v2i2.520

86. Rey Casal C. Ensayo del etileno y de una citoquinina como posibles fitosanitarios frente a Botrytis cinerea en la plantación ornamental de Zinnia elegans. [tesis maestría]. [Coruña]: Universidad de Cataluña; 2016. [citado 26 de febrero de 2020]. Recuperado a partir de: https://ruc. udc.es/ dspace/bitstream/handle/2183/16149/ReyCasal_Carlota_TFM_2016.pdf?sequence=2\&is Allowed $=\mathrm{y}$

87. Olivera Gonzales P, Espinoza del Rio R, Tamariz Angeles C. Multiplicación in vitro y embriogénesis somática de Perezia pinnatifida (Asteraceae) planta medicinal andina. Rev Peru Biol 2017;24(3):323-8. DOI: https://doi.org/10.15381 /rpb.v24i3.13911

88. Carranza C, Castellanos G, Deaza D, Miranda D. Efecto de la aplicación de reguladores de crecimiento sobre la germinación de badea (Passiflora quadrangularis) en condiciones de invernaderos. Rev Colomb Cienc Hortic 2016;10(2):284-91. DOI: https://doi.org/10.17584/rcch.2016v10i2. $\underline{5791}$
89. Yashwanti Y, Sharma MK, Singh D, Kumar K, Verma AK. Effects of auxins and cytokinin on budding and growth of saplings of bael (Aegle marmelos Correa) a medicinal plant. J Agric Ecol 2017;3:12-8.

90. Shams M, Yildirim E, Ekinci M, Agar G, Turán M, Kul R. Exogenous cytokinin application increased the capsaicin and ascorbic acid content in pepper fruit. Scientific Papers. Series B, Horticulture 2018;67:507-11.

91.Basra SMA, Lovatt CJ. Exogenous applications of Moringa leaf extract and cytokinins improve plant growth, yield and fruit quality of cherry tomato. Horttechnology 2016;26(3):327-37. DOI: https://doi.org/10.21273/HORTTECH.26.3.327

92.Bagahawatta A, Kumara K. Application of cytokinin and gibberellic acid to regulate flower quality in Dendrobium orchid. In: Bagahawatta A, Kumara K, editors. Proceeding - $20174^{\text {th }}$ International conference on Agriculture and Forestry 2017 [Internet]. Sri Lanka Technological Campus, Sri Lanka; 2018. p. 1-5. DOI: https://doi.org/ 10.17501/icoaf.2017.3201

93. Suwandi T, Dewi K, Cahyono P. Pineapple harvest index and fruit quality improvement by application of gibberellin and cytokinin. Fruits 2016;71(4):209-14. DOI: https://doi.org/10.1051/ fruits/2016010

94.Di Benedetto A, Galmarini C, Tognetti JA. Exogenous cytokinin promotes Epipremnum aureum $\mathrm{L}$. growth through enhanced dry weight assimilation rather than through changes in partitioning. Am J Exp Agric 2014;5(5):419-39. DOI: https://doi.org/10.9734/AJEA/2015/13398

Nota del Editor:

Journal of the Selva Andina Biosphere (JSAB) se mantiene neutral con respecto a los reclamos jurisdiccionales publicados en mapas y afiliaciones institucionales. 